\title{
NURBS based B-rep Models for Macromolecules and their Properties
}

\author{
C.Bajaj H.Y.Lee R.Merkert V.Pascucci \\ Department of Computer Sciences, \\ Purdue University, \\ West Lafayette, Indiana
}

\section{Introduction}

We consider NURBS based data structures for molecules and their properties, to support synthetic drug design and structural reasoning applications in molecular chemistry. The difficulty of modeling and visualization of large molecules derives from the high combinatorial complexity of the typical molecule considered (e.g. proteins or nucleic acids [6, 12]). There are two main modeling approaches. The first describes the molecule's primary structure and the detailed 3D position of each of its atoms. The second groups some regions of the molecule into simpler shapes to describe the folding of the molecule into its secondary, tertiary and higher order structures.

In this paper we focus on the first case where it is required to represent the primary structure of a macromolecule. One representation of the primary structure is the space flling model, where each atom is described by a sphere with its van der Waals radius. The topological and combinatorial structure of this model has recently been explored by Edelsbrunner [9, 10, 11]. In particular from such formalization any information regarding the topological structure and the corresponding geometry of the molecule can be easily extracted.

On the basis of such results we develop a B-rep data structure that aims to be useful both for visualization and modeling purposes. This requires the ability (a) to exactly represent the shape of the molecule, (b) to directly render such a representation, and (c) to perform modeling operations that correspond to the addition/deletion of residues. The natural choice to achieve both goals is to use trimmed NURBS (Non Uniform Rational tensor-product B-Spline with rational B-Spline trimming curves). They are an industry-wide standard as a modeling primitive and graphics libraries for
NURBS rendering are available (e.g. openGL, OpenInventor [18]). Moreover, the rational parameterization allows for an exact representation of a spherical surface. This alone is not sufficient. In order to have an exact representation of a macromolecular structure we also need to represent for each atom, not its entire sphere, only that portion of the sphere which belongs to the external molecule surface. This means that from the sphere which represents one atom we must cut away the pieces contained in the neighboring atoms. We can prove that adopting a certain parameterization each trimming curve (a circle) in the $3 \mathrm{D}$ space is mapped back in the parameter domain to a curve that can be in turn represented exactly as a NURBS curve. In this way we can represent the contribution of each atom to the molecule surface with a trimmed NURBS patch without any approximation.

The method applies for example to the determination of the solvent accessible surface (the offset of a union of balls is a union of balls) and generalizes to achieve the exact representation of the solvent contact surface (also known as the Connolly surface of the molecule $[7,8]$ ).

The main contributions of the paper are: (a) the definition of a (minimal size) B-rep with standard trimmed NURBS representation (section 3), (b) parametric Brep model of the solvent accessible surface useful for animation (section 4), (c) the classification of the solvent contact surface and computation of its representation as a trimmed NURBS.

\section{Boundary Representation of Molecule (Property) Surfaces}

The representation we use for molecule (property) surfaces is a boundary representation. Two classes of information are used: (a) geometric description of each patch, (b) topological relations amongst the patches. We maintain the following data structures related to the molecule. 
1. The weighted Voronoi diagram $[1,14]$ (power diagram) $\mathcal{D}$ of the molecule atom centers (the weights are the squares of the atoms radii).

2. A regular triangulation $\mathcal{T}$ (dual of the power diagram) of the same set of weighted points as in [11].

3. A NURBS patch per molecule atom.

As it will be shown in the following sections these data structures contain all the relevant information for the molecule surface. Edelsbrunner [9, 10, 11] has shown that the intersections among atoms in a molecule correspond to some edges of the regular triangulation $\mathcal{T}$ (those in the $\alpha=0$ shape). This implies that the edges of a specific subgraph of the regular triangulation correspond also to adjacency relations between boundary patches of the solvent accessible surface. Similarly, we have that the adjacency relations between pairs of spherical or toroidal patches in the solvent contact surface correspond to incidence relations (vertex-edge or edge-facet) of the boundary of the corresponding regular triangulation. Hence the $\alpha=0$ shape provide us with topological relations between the patches that form the molecular surface.

The power diagram provides us with the cutting planes that generate each spherical patch. This enables us to determine the exact representation of the molecule surface. By translating the same planes we get the cutting planes necessary to determine the exact description of the solvent contact surface.

The NURBS patches are thus defined in term of these two data structures. We are able to update them dynamically as we change a parameter (e.g. the radius of the solvent atom or the number of atoms in the molecule) of the representation to have an efficient representation. This yields a parametric B-rep of the solvent accessible/contact surface for animation.

\section{Atom Boundary Patch as a Trimmed NURBS}

As we have outlined in the introduction we have selected NURBS as basic modeling primitive [15]. In this section we present the method for computing an exact NURBS representation for the spherical patches that compose the external surface of a molecule. This differs from the work of Piegl [17], where the goal was to represent an entire sphere as piecewise rational Bezier or NURBS patch. In particular, for an appropriate choice of parameterization we obtain a single trimmed NURBS for each atom's external surface contribution. Each such patch is the intersection of one sphere (representing one atom) with the exterior of all its neighboring spheres. Consider the intersection $S \cap R$ of a spherical surface $S=\left\{x:\left\|x-x_{0}\right\|=r^{0}\right\}$ with the external of sphere
$R=\left\{x:\left\|x-x_{1}\right\| \geq r^{1}\right\}$. There always exists an halfspace $\pi=\{x:(x \cdot l) \leq d\}$ such that:

$$
S \cap R=S \cap \pi
$$

For each atom we can reduce our patch representation problem to the intersection of a sphere with a set of halfspaces. The union of balls model [9] provides the equation of each halfspace intersecting one atom ${ }^{1}$. Note that, since we use a parametric representation $S=f(u, v)$, we need to compute the domain $D$ in $(u, v)$ space such that $f(D)=S \cap \pi$.

To have an efficient representation we want to obtain only one NURBS patch per atom. Moreover, since we will use this formulation to achieve a representation of the surface parametric in the radii of the atoms we need a formulation that maps continuous modifications of the radii into continuous modifications of the domain $D$. This is not achieved with the classical NURBS sphere representation as a rotational surface of a half circle [17] since there are two points (north and south pole) of the sphere that are the image of two lines in the parameter domain (say $u=0, u=1$ if the interval of the $u$ domain is $[0,1])$. This implies that when the boundary plane of $\pi$ crosses one of the poles the corresponding trimming curve in the $(u, v)$ domain would have a discontinuous change in shape.

Without loss of generality we assume that $S$ is the unitary sphere. The parameterization we adopt is the following (see [4]):

$$
\begin{aligned}
& x=\frac{2 u}{u^{2}+v^{2}+1} \\
& y=\frac{2 v}{u^{2}+v^{2}+1} \\
& z=\frac{u^{2}+v^{2}-1}{u^{2}+v^{2}+1}
\end{aligned}
$$

This parameterization maps the (infinite) rectangular domain

$$
[-\infty,+\infty] \times[-\infty,+\infty]
$$

to the unitary sphere. Note that in practice we do not deal with an infinite domain since we do not represent an entire sphere but only one spherical patch. In particular assume that we are considering the intersection $S^{*}$ of the unit sphere $S$ with the halfspace $z \leq d$ (with a rigid body transformation and a scaling we can always reduce the first intersection to this case). We determine a positive constant $l$ such that $S^{*} \subset f(I)$, where $I$ is the square domain $[-l,+l] \times[-l,+l]$. In the parameter domain this corresponds to the condition $D \subset I$. The minimum value of $l$ that satisfies such condition

\footnotetext{
${ }^{1}$ Given the Voronoi complex of the weighted centers of the molecule atoms, the halfspaces whose common intersection generates the Voronoi cell of the atom $B$ are those with which $S=\partial B$ must be intersected.
} 
is $l=\sqrt{\frac{1+d}{1-d}}$. Regarding the numerical stability of the method it is important to note that for $d=0.999$ we get $l=44.710 \ldots$. Even when $d$ is much larger than a realistic value, we still deal with a small domain region.

The next step is to determine the domain $D$. At this end we simply replace the parametric equations (1) of the of the sphere to the variables in the Cartesian inequality of $\pi$ obtaining the Cartesian inequality defining D.

$$
\frac{u^{2}+v^{2}-1}{u^{2}+v^{2}+1} \leq d \quad \Rightarrow \quad u^{2}+v^{2} \leq l^{2}
$$

Thus the domain $D$ is a disc with center in the origin and radius $l$. Note that a variation of $d$ corresponds to a scaling of $D$, that can be performed by simply scaling its control polygon (once a NURBS representation is defined for the trimming curve of $D$ ). For any additional cutting halfspace $\bar{\pi}: a x+b y+c z \leq d$ we have:

$$
(c-d) u^{2}+(c-d) v^{2}+2 a u+2 b v-(c+d) \leq 0
$$

If the plane $a x+b y+c z=d$ contains the singular point of the parameterization $P=(0,0,1)$ then $c=d$. In this case the trimming curve is the straight line:

$$
2 a u+2 b v-(c+d)=0 .
$$

The domain $D$ must be intersected with the half-plane $2 a u+2 b v-(c+d) \leq 0$.

If $c-d \neq 0$ the trimming curve derived from (3) has Cartesian equation:

$$
\left(u+\frac{a}{c-d}\right)^{2}+\left(v+\frac{b}{c-d}\right)^{2}=\frac{a^{2}+b^{2}+c^{2}-d^{2}}{(c-d)^{2}}
$$

In general we note that all the trimming curves are circles (possibly with infinite radius) so that the region $D$ can be modeled as progressive intersection/difference of a sequence of circles. Corresponding to the cutting halfspace $\bar{\pi}$ of normalized equation $a x+b y+c z \leq d$, with $a^{2}+b^{2}+c^{2}=1$, we have in parameter space a circle $C$ of center $\left(\frac{a}{c-d}, \frac{b}{c-d}\right)$ and radius $\frac{\sqrt{1-d^{2}}}{c-d}$. The region defined by such circle (inside/outside) depends on the sign of the term $c-d$. For $c-d<0 P=(0,0,1)$ is inside $\bar{\pi}$ and hence the points of the plane at infinity are included in the region corresponding to $\bar{\pi}$. That is $\bar{\pi}$ is mapped onto the outside of $C$. This requires $C$ to be parameterized with a clockwise orientation. Symmetrically $c+d<0$ implies that $\bar{\pi}$ corresponds to the region inside $C$ and hence $C$ must be parameterized with a counterclockwise orientation.

In appendix A we detail the control points computation used for the definition of the spherical patch.

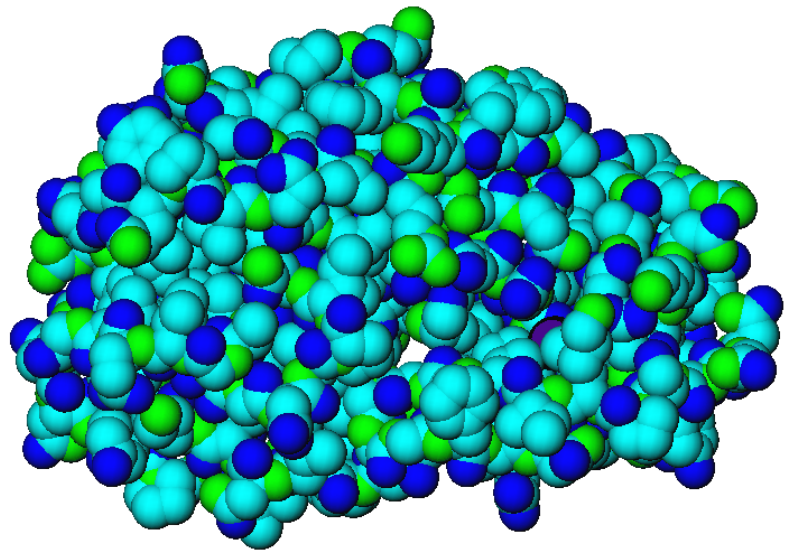

(a)

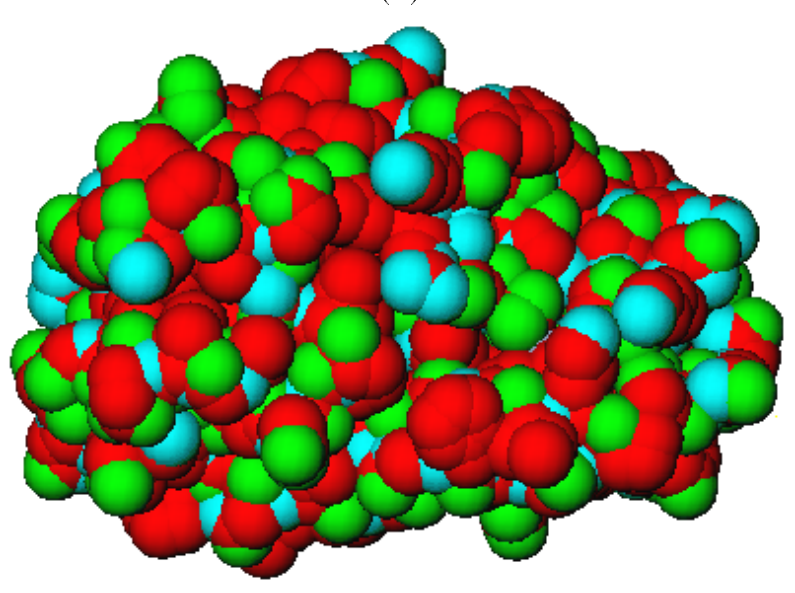

(b)

Figure 1: The HIV-2 PROTEASE (a) and one solvent accessible surface (b) for the same molecule.

\section{Solvent Accessible Surface}

In this section we discuss the representation of the solvent accessible surface of a molecule. Since we are representing the molecule with a union of balls $\mathcal{B}$, in the following, with some abuse of terminology, we will call $\mathcal{B}$ both the molecule or the union of balls. Similarly each single ball $B$ will be called either a ball or an atom.

Assume we have a ball $B$ of radius $r$ (a solvent atom) free to move in space without intersecting the union of balls $\mathcal{B}$ (a molecule). We say that $B$ is in a legal position if its interior $\stackrel{\circ}{B}$ does not intersect $\mathcal{B}$.

Definition 1 The solvent accessible surface $S_{a}$ of the union of balls $\mathcal{B}$ relative to a solvent atom $B$ of radius $r$, is the locus (envelope) of the centers of the spheres with radius $r$ tangent to $\mathcal{B}$.

From $[9,10]$ we know that $S_{a}$ is the boundary surface of the union of balls $\mathcal{B}^{\prime}$ that has the same set of atoms as $\mathcal{B}$ but with all the radii increased by $r$ (see figure 1 ). On the basis of this property we can achieve a repre- 
sentation of $S_{a}$ parametric in $r$. For $r=0$ we obtain the van der Waals surface of the molecule $\partial \mathcal{B}$. Varying the value of $r$ we get the accessible surfaces of different solvents.

Let $V^{\prime} \in \mathcal{V}^{\prime}$ be the convex cell corresponding to the ball $B^{\prime} \in \mathcal{B}^{\prime}$. $V^{\prime}$ is the intersection of a set of $k$ halfspace $\pi_{1} \cap \ldots \cap \pi_{k}$. The the contribution of $B$ to the boundary of $\mathcal{B}$ (the surface $S_{a}$ for $r=0$ ) is given by $\partial B \cap \pi_{1} \cap$ $\ldots \cap \pi_{k}$.

Now assume $r>0$ and consider the sphere $B^{\prime}$ in $\mathcal{B}^{\prime}$ corresponding to $B$ in $\mathcal{B}$. The contribution of $B^{\prime}$ to $S_{a}$ is computed by intersecting $\partial B^{\prime}$ with the same set of halfspaces $\pi_{1}^{\prime}, \ldots, \pi_{k}^{\prime}$.

To compute the trimming curves in the parameter space $(u, v)$ of the NURBS patch representing $\partial B^{\prime} \cap \pi_{1}^{\prime} \cap$ $\ldots \cap \pi_{k}^{\prime}$, we apply a mapping that transforms $B^{\prime}$ into the unitary ball $B_{u}$. Under this mapping the variation of $r$ corresponds to have a fixed (unitary) radius ball $B_{u}$ intersected with a set of varying halfspaces. Formally, if the ball $B^{\prime}$ and one halfspace $\pi^{\prime}$ have equations:

$$
\begin{array}{cc}
B^{\prime}: & x^{2}+y^{2}+z^{2} \leq R^{2} \\
\pi^{\prime}: & a x+b y+c z+d \leq 0
\end{array}
$$

we apply the coordinate transformation $x=R x^{\prime}, y=$ $R y^{\prime}, z=R z^{\prime}$ to map $B^{\prime}$ to $B_{u}$ :

$$
\begin{array}{cc}
B_{u}: & x^{\prime 2}+y^{2}+z^{2} \leq 1 \\
\pi^{\prime}: & a x^{\prime}+b y^{\prime}+c z^{\prime}+\frac{d}{R} \leq 0
\end{array}
$$

The change of the radius $R$ of $B^{\prime}$ to $R+r$ is hence mapped in normalized coordinates $\left(x^{\prime}, y^{\prime}, z^{\prime}\right)$ to the change of the parameter $\frac{d}{R}$ of the halfspace $\pi^{\prime}$ to $\frac{d}{R+r}$. This means that the equation of the trimming circles can be rewritten, including the parameter $r$, as:

$\left(u+\frac{a}{c-\frac{d}{R+r}}\right)^{2}+\left(v+\frac{b}{c-\frac{d}{R+r}}\right)^{2}=\frac{a^{2}+b^{2}+c^{2}-\left(\frac{d}{R+r}\right)^{2}}{\left(c-\frac{d}{R+r}\right)^{2}}$

that is a circle of center $\left(\frac{a}{\frac{d}{\pi+r}-c}, \frac{b}{\frac{d}{\pi+r}-c}\right)$ and radius $r=$ $\frac{\sqrt{(R+r)^{2}-d^{2}}}{|(R+r) c-d|}$. To maintain the description of the domain $D$ we have to maintain a $2 \mathrm{D}$ dynamic union of balls that is equivalent to maintain a weighted Voronoi diagram of moving points in the plane $[13,5]$.

Note also that the coefficient $d$ of the plane equation is also function of $r$. In fact as the radius of each ball is increased by $r$ the Voronoi plane that separates two balls moves toward the smaller one. An example is shown in figure 2. The distances $l_{1}, l_{2}$ of the Voronoi plane $\pi$ from the centers of the two balls must be such that the power distances of $\pi$ are equal, that is:

$$
l_{1}^{2}-r_{1}^{2}=l_{2}^{2}-r_{2}^{2}
$$

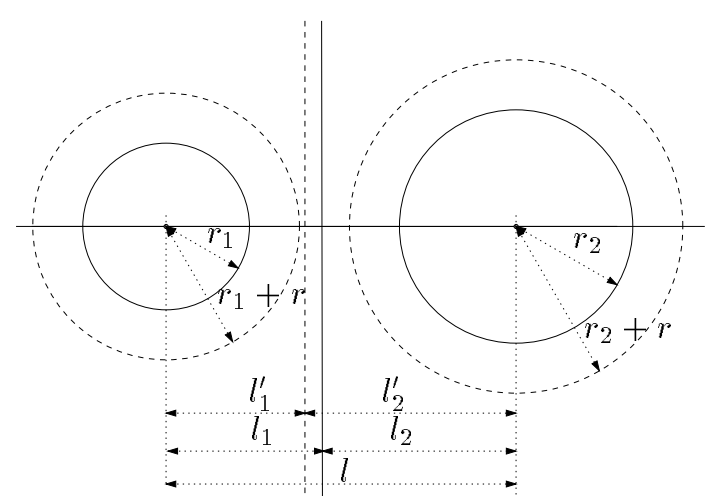

Figure 2: As the radius of the two balls is increased by 1 the Voronoi plane that separate them moves towards the smaller ball.

Moreover the sum of two distances is constant (the two balls grow but do not move):

$$
l_{1}+l_{2}=l
$$

From these two equations we get for $l_{1}$ so:

$$
l_{1}^{2}-r_{1}^{2}=\left(l-l_{1}\right)^{2}-r_{2}^{2} \Rightarrow l_{1}=\frac{l^{2}+r_{1}^{2}-r_{2}^{2}}{2 l}
$$

When $r_{1}$ changes to $r_{1}+r$ and $r_{2}$ changes to $r_{2}+r$ we have:

$$
\begin{aligned}
& l_{1}^{\prime}=l_{1}+\frac{r_{1}-r_{2}}{2 l} r \\
& l_{2}^{\prime}=l_{2}+\frac{r_{2}-r_{1}}{2 l} r
\end{aligned}
$$

\section{$5 \quad$ Rolling Ball Surface}

In this section we extend the method to achieve an exact NURBS representation of the rolling ball surface $S_{r}$ of a molecule $\mathcal{B}$. The goal is to achieve an intermediate stage toward to construction the solvent contact surface $S_{c}$ defined in the next section. In Figure 3 is shown the Fullerene molecule along with two solvent contact surfaces corresponding to two different solvent radii.

We assume to have a ball $B$ of radius $r$ (the solvent molecule) which is free to roll along the union of balls $\mathcal{B}$ (the molecule). The union of all the balls $B$ (moving tangentially to $\mathcal{B}$ in all the possible directions) is a region whose outer envelope strictly contains (if $r>0$ ) $\mathcal{B}$ and whose inner envelope is tangent to $\mathcal{B}$ (see [3]).

Definition 2 The rolling ball surface $S_{r}$ of the molecule $\mathcal{B}$ with respect to a ball $B$ of radius $r$ is the inner envelope of the region described by $B$ rolling on $\mathcal{B}$ in all possible directions.

The close relationship between the solvent accessible surface and the rolling ball surface is evident from this definition. 


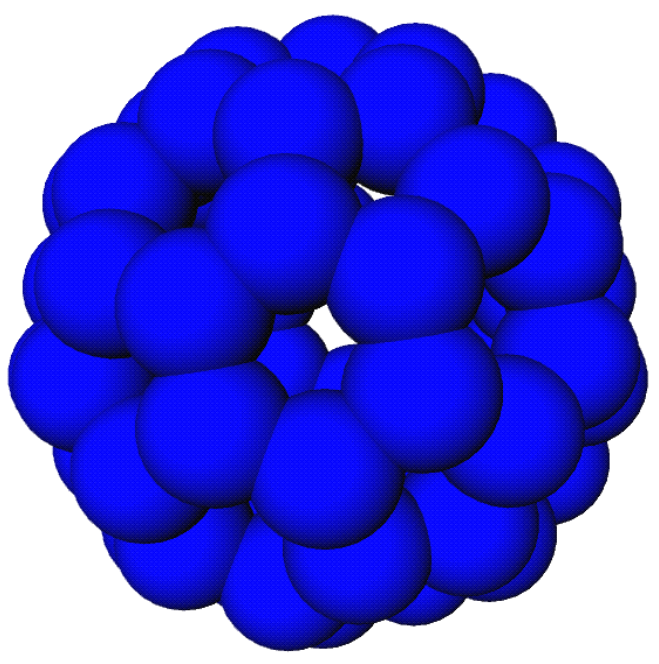

(a)

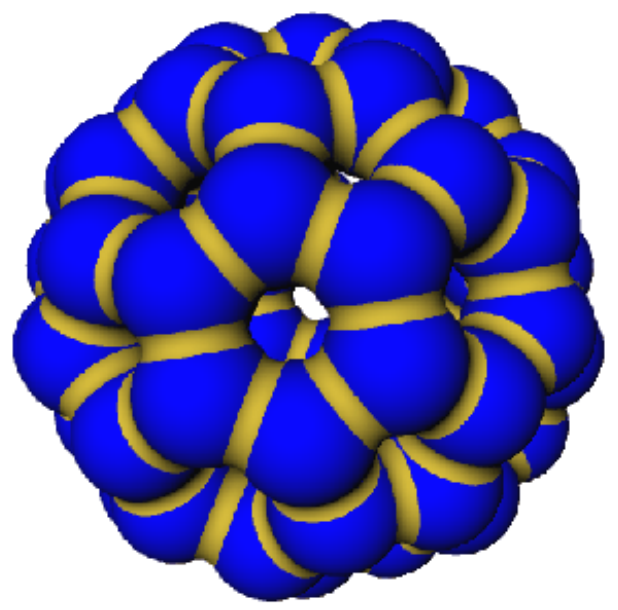

(b)

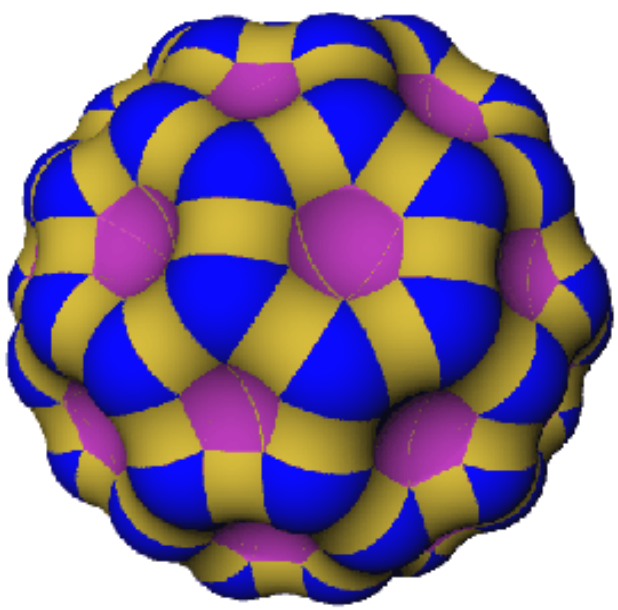

(c)

Figure 3: The Fullerene molecule (a) and two solvent contact surfaces (b),(c) corresponding to two different solvent radii.
Lemma 1 (Necessary Condition) If a point $p$ lies on the rolling ball surface $S_{r}$ then it lies also on the boundary of a ball $B$ with center on the solvent accessible surface $S_{a}$.

Proof: By Definition 2 when $p \in S_{r}$ there exists a ball $B$ of radius $r$ such that $p \in \partial B, B \cap \mathcal{B} \neq \emptyset$ and $\stackrel{\circ}{B} \cap \mathcal{B}=\emptyset$. But if the center $q$ of $B$ does not belong to $S_{a}$ either

$$
B \cap \mathcal{B}=\emptyset
$$

or

$$
\stackrel{\circ}{B} \cap \mathcal{B} \neq \emptyset
$$

Using the regular triangulation $\mathcal{T}^{\prime}$ associated with $\mathcal{B}^{\prime}$ we can define the set of patches composing $S_{r}$. First, recall the relationship between $\partial \mathcal{T}^{\prime}$ and $\partial \mathcal{B}^{\prime}$ :

- each vertex $v$ of $\partial \mathcal{T}^{\prime}$ corresponds to a spherical patch of $\partial \mathcal{B}^{\prime}$;

- each edge $e$ of $\partial \mathcal{T}^{\prime}$ corresponds to the intersection line between two adjacent spherical patches of $\partial \mathcal{B}^{\prime}$;

- each triangle $t$ of $\partial \mathcal{T}^{\prime}$ corresponds to the intersection point between three adjacent spherical patches of $\partial \mathcal{B}^{\prime}$.

We base the construction of the rolling ball surface on these properties. Using Connolly's terminology [7] (as we will later see that the solvent contact surface is a subset of the rolling ball surface) we have (a) each vertex $v$ of $\partial \mathcal{T}$ ' corresponding to a "convex" spherical patch in $S_{r}$, (b) each edge $e$ of $\partial \mathcal{T}^{\prime}$ corresponding to a "saddle" toroidal patch in $S_{r}$, and (c) each triangle $t$ of $\partial \mathcal{T}$ ' corresponding to a "concave" spherical patch in $S_{r}$. The definitions of these three kinds of patches are reported in the following three subsections.

\section{1 "Convex" Spherical Patches}

Consider the spherical patch $\bar{v}$ with radius $r+r_{1}$ of $\partial \mathcal{B}^{\prime}$ associated with the vertex $v$ (see figure 4 ). It represents a moving solvent ball that maintains contact with $\partial \mathcal{B}$ at a point $p$. The surface described by the point $p$ is in turn a spherical patch of radius $r$ (part of $\partial \mathcal{B})$. It can be computed from the power diagram of $\partial \mathcal{B}$. Call $B$ the ball (of radius $r_{1}$ ) of $\mathcal{B}$ with center $v$. It contributes the patch $\partial B \cap \pi_{1} \cap \ldots \cap \pi_{k}$ (that is the Voronoi cell of $v$ is $\left.\pi_{1} \cap \ldots \cap \pi_{k}\right)$ to $\partial \mathcal{B}$. The ball $B$ contributes the patch $\partial B \cap \bar{\pi}_{1} \cap \ldots \cap \bar{\pi}_{k}$ to $S_{e}$, where $\bar{\pi}_{i}$ is parallel to $\pi_{i}$ but nearer to $v$. Without loss of generality we assume $v$ to be the origin $(0,0,0)$ and $\pi_{1}$ to be orthogonal to the $x$ axis (with a rigid body transformation we can always achieve this situation). The halfspace $\pi_{1}$ is $x \leq d$ and the halfspace $\bar{\pi}_{1}$ is $x \leq \bar{d}$ where: 

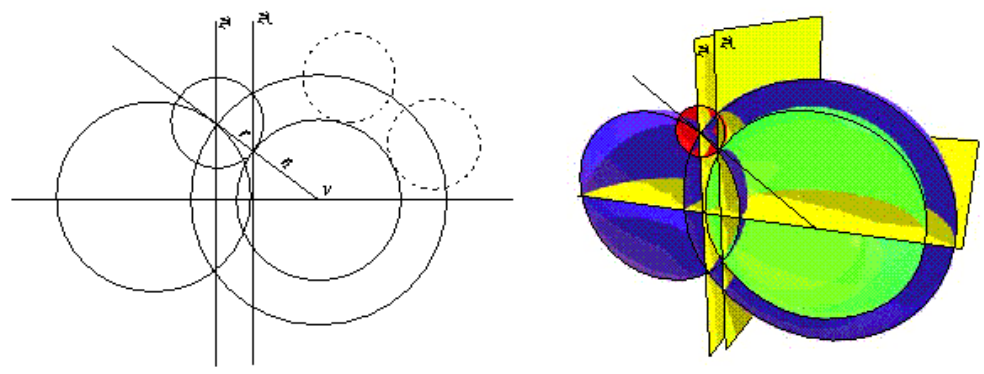

Figure 4: A solvent atom of radius $r$ that rolls on the molecule surface $\mathcal{B}$ maintaining its center on the solvent accessible surface $\mathcal{B}^{\prime}$. Its point of contact with $\mathcal{B}$ belongs to the solvent contact surface $S_{c}$.

$$
\bar{d}=\frac{d r_{1}}{\left(r+r_{1}\right)}
$$

We can so determine any halfspace $\bar{\pi}_{i}$ corresponding to $\pi_{i}$ and hence $\partial B \cap \bar{\pi}_{1} \cap \ldots \cap \bar{\pi}_{k}$.

\section{2 "Saddle" toroidal patches}

A similar argument holds for saddle toroidal patches. With reference to figure 5 we consider the edge $e$ of $\partial \mathcal{T}^{\prime}$ with extreme vertices $v_{1}$ and $v_{2}$. The edge $e$ corresponds on $\partial \mathcal{B}^{\prime}$ to a (portion of) circle $\bar{e}$ of intersection between two adjacent balls $\partial B_{1} \cap \partial B_{2}$. Thus, it is possible to roll a solvent ball, moving its center along the arc $\bar{e}$.

If the edge $e$ is not a facet of any triangle of $\partial \mathcal{T}^{\prime}$ then $\bar{e}$ is an entire circle. The ball that rolls maintaining its center on $\bar{e}$ describes a torus $E$. We are interested in just a portion of $\partial E$. Consider the plane $\pi$ of the Voronoi diagram on which $e$ lies. Applying the procedure specified in the previous subsection we compute two planes $\pi_{1}$ and $\pi_{2}$ by translating $\pi$ towards $v_{1}$ and $v_{2}$, respectively. The intersection of $\partial E$ with the region within $\pi_{1}$ and $\pi_{2}$ generates two toroidal patches. The one nearest to the torus axis $\overline{v_{1} v_{2}}$ is the toroidal patch $E^{*}$ that belongs to $S_{r}$.

If the edge $e$ is the arc from point $\bar{t}_{1}$ to point $\bar{t}_{2}$ then the toroidal patch associated with $e$ is the portion of the patch $E^{*}$ intersected with two more halfspaces. Call $\pi\left(v_{1}, v_{2}, v_{3} ; v_{4}\right)$ the halfspace that contains $v_{1}, v_{2}, v_{3}$ in its boundary and $v_{4}$ in its interior (with $v_{1}, v_{2}, v_{3}, v_{4}$ affinely independent). The toroidal patch corresponding to $e$ is (see figure 5 ):

$$
E^{*} \cap \pi\left(v_{1}, v_{2}, \bar{t}_{1} ; \bar{t}_{2}\right) \cap \pi\left(v_{1}, v_{2}, \bar{t}_{2} ; \bar{t}_{1}\right) .
$$

\section{3 "concave" spherical patches}

Finally, consider the triangle $t$ of $\partial \mathcal{T}^{\prime}$ with vertices $v_{1}$, $v_{2}$, and $v_{3}$. It corresponds to the point $\bar{t}$ in $\partial \mathcal{B}^{\prime}$. In
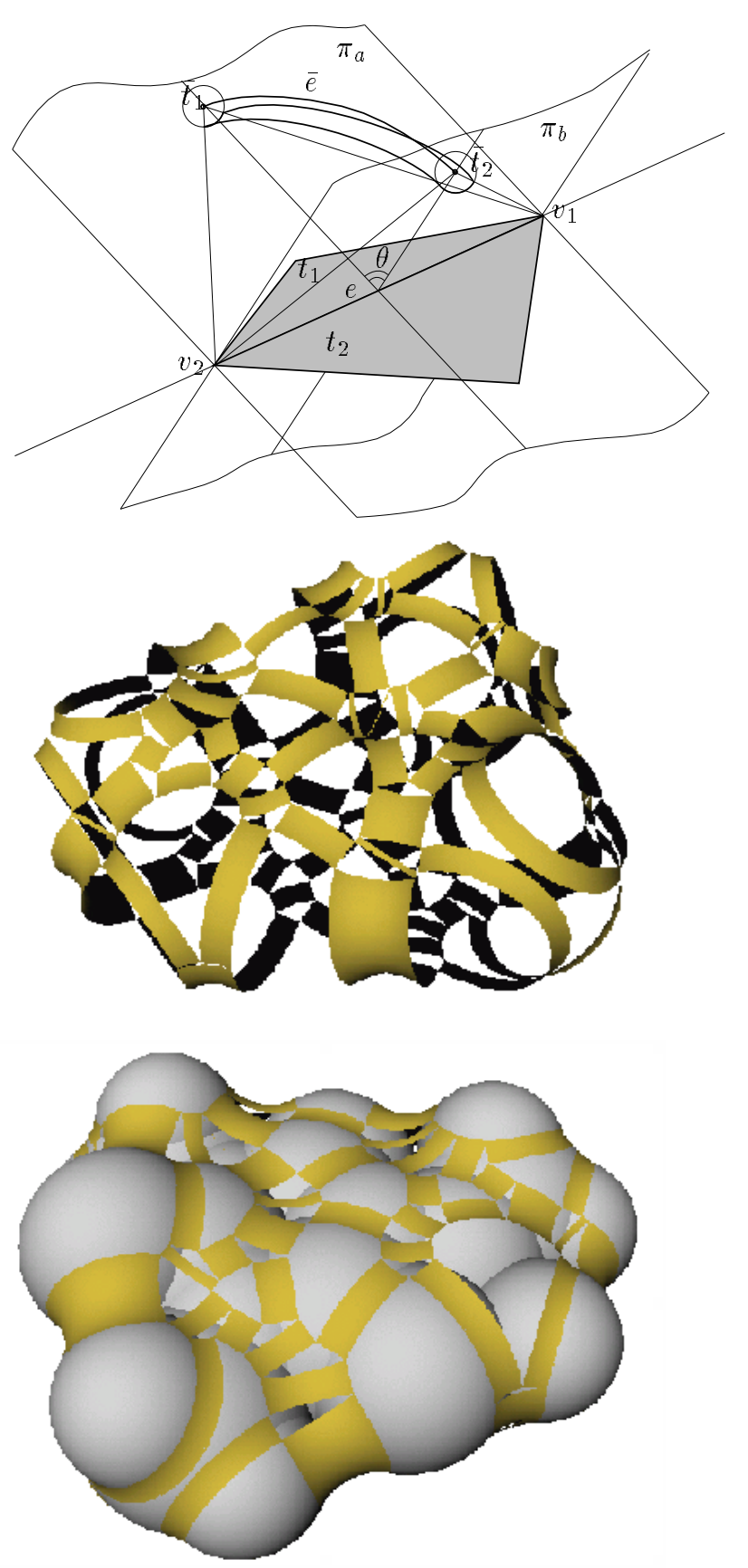

Figure 5: (Top) A solvent atom $B$ of radius $r$ that rolls on the molecule surface $\mathcal{B}$ maintaining its center on the solvent accessible surface $\mathcal{B}^{\prime}$ and two points of contact with two molecule atoms. The portion of circle of $\partial B$ that belongs to the triangle with the three vertices $v_{1}, v_{2}$ center of $B$, belongs to the rolling ball surface $S_{r}$. (Middle) The toroidal NURBS patches of the rolling ball surface $S_{r}$ of the caffeine molecule. (Bottom) The toroidal NURBS patches of $S_{r}$ shown together with the union of balls. 


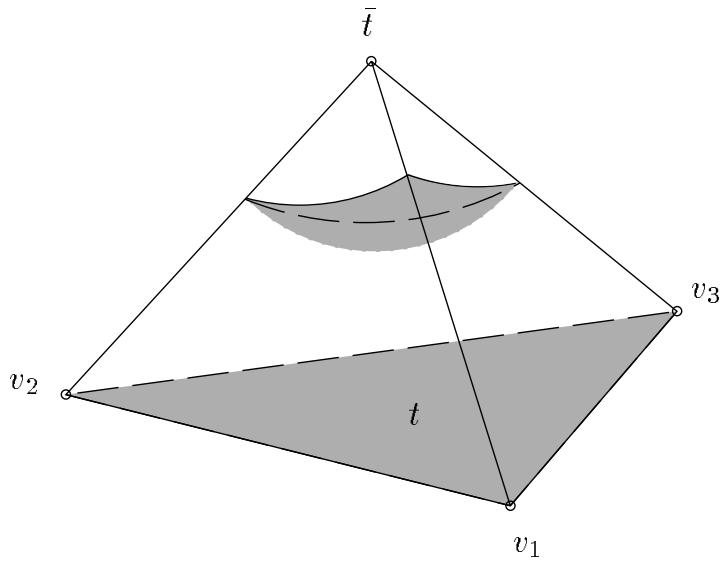

Figure 6: A solvent atom of radius $r$ tangent to the molecule surface $\mathcal{B}$ maintaining its center on the solvent accessible surface $\mathcal{B}^{\prime}$ and three points of contact with three molecule atoms. The portion of $\partial B$ inside the tetrahedron with vertices $v_{1}, v_{2}, v_{2}$, center of $B$, belongs to the rolling ball surface $S_{c}$.

this case we have a solvent atom $B$ with no degrees of freedom (it cannot roll since its center is fixed in $\bar{t}$ ). The contribution of $B$ to $S_{c}$ is thus given by:

$$
\partial B \cap \pi\left(v_{1}, v_{2}, t ; v_{3}\right) \cap \pi\left(v_{1}, v_{3}, t ; v_{2}\right) \cap \pi\left(v_{2}, v_{3}, t ; v_{1}\right)
$$

Figure 7 depicts a complete solvent contact surface (a superset of the rolling ball surface) of the caffeine molecule with the concave patches highlighted in purple.

\subsection{Correctness of the Result}

In this subsection we show that the set of patches we have computed form the required rolling ball surface $S_{r}$.

We have already shown in Lemma 1 that any point $p$ of $S_{r}$ must belong to the boundary of a ball $B$ whose center belongs to $\partial \mathcal{B}^{\prime}$.

Take the point $p$ in $\partial B$ and the direction $\vec{d}=\overrightarrow{o p}$ where $o$ is the center of $B$ (with $o \in \partial \boldsymbol{B}^{\prime}$ ). It easy to verify that $p \in S_{r}$ iff the following property holds.

Property 1 Given a direction of translation $\overrightarrow{d^{*}}$ iff $\vec{d} \cdot \overrightarrow{d^{*}}>0$ there is always a small $\delta>0$ such that any translation of $B$ of an $\epsilon>0$ with $\epsilon<\delta$ along the direction $\overrightarrow{d^{*}}$ will intersect $\mathcal{B}$.

Theorem 1 Any point in the rolling ball surface of the molecule $\mathcal{B}$ belongs to one of the patches of $S_{r}$ computed in the section above.

Proof: From Lemma 1 we have that all the points $p$ of $S_{r}$ belong to the boundary of some tangent solvent ball $B$. Also for each solvent ball $B$ of radius $r$ its intersection points $p$ with $S_{r}$ are (all) its boundary points for
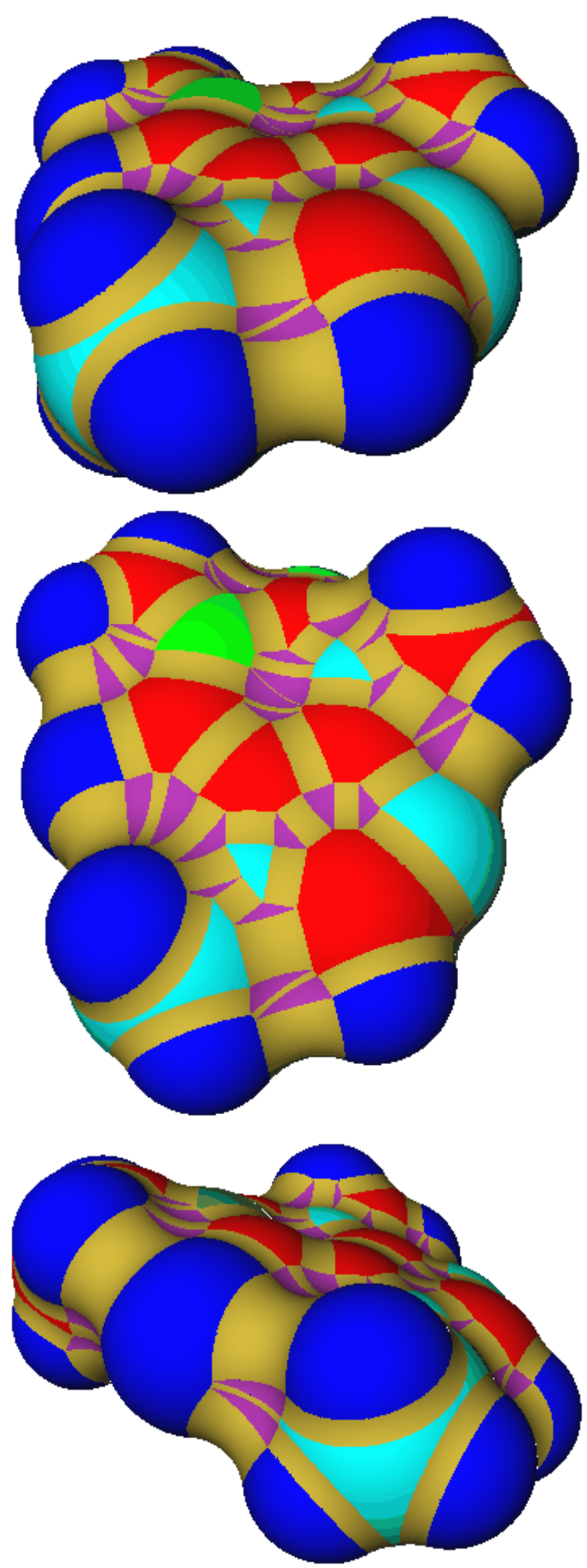

Figure 7: Complete Connolly surface of a caffeine molecule. 
which property 1 holds. Thus, the fact that there is a small neighborhood of $B$ where no legal ball $B^{\prime}$ contains in its interior any point $p \in S_{r}$, proves the theorem. $\diamond$

\section{Solvent contact surface}

In this section we extend the method to achieve an exact NURBS representation of the solvent contact surface $S_{c}$ (also known as the Connolly surface) of a molecule $\mathcal{B}$. The surface is defined as follows.

Definition 3 A point $p$ belongs to the solvent contact surface $S_{c}$ of the molecule $\mathcal{B}$ with respect to a solvent with atoms of radius $r$ iff:

- there exists a legal ball $B_{1}$ of radius $r$ that contains $p$ in its boundary:

$$
\exists B_{1} \mid p \in \partial B_{1} \text { and } \stackrel{\circ}{B}_{1} \cap \mathcal{B}=\emptyset
$$

- there is no legal ball $B_{2}$ of radius $r$ that contains $p$ in its interior:

$$
\stackrel{\circ}{B}_{2} \cap \mathcal{B}=\emptyset \quad \Rightarrow \quad p \notin \stackrel{\circ}{B} 2
$$

The close relationship between the solvent contact surface and the rolling ball surface becomes clear from this definition.

Lemma 2 If a point $p$ lies on the solvent accessible surface $S_{c}$ then it lies also on the rolling ball surface $S_{r}$.

Proof: The proof can immediately be derived from the comparison of definition 2 with definition 3. Further, from this follows that lemma 1 holds not only for $S_{r}$, but also for $S_{c}$.

The problem that remains to be solved is the removal of (possible) self intersections that the rolling ball surface might have, and that make it differ from the solvent contact surface (for a classification of the classes of selfintersection that may occur see [3], fig1). This problem can be geometrically highlighted even with a set of two small balls along which a large radius probe is rolled (see figure 8 ). In this case the blending surface is formed by a toroidal patch that is self-intersecting.

To show the same problem for the concave patches at least three spheres are needed. Figure 9 shows three possible configurations of the solvent contact surface for a set of three balls. From the picture it is clear how complex the shape can get (with sharp features, varying in genus and possibly disconnected) even for a simple configuration of three balls.

In the following subsections we will show how the patches of the rolling ball surface can be trimmed to get the exact representation of the solvent contact surface. As for the previous case we will report a brief sketch of the proof of correctness.

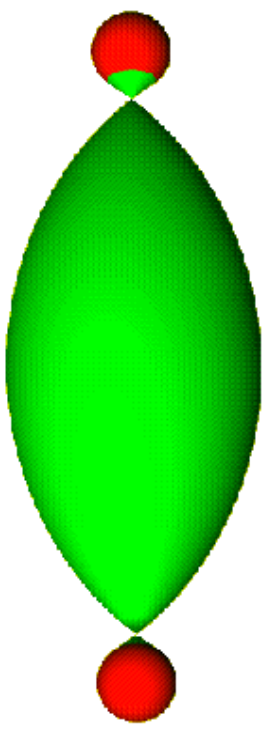

(a)

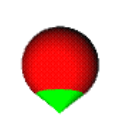

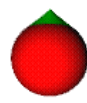

(b)
Figure 8: (a) The rolling ball surface (in green) with a probe of radius 10 on two spheres (in red) of radius 1 is a self intersecting surface. (b) The corresponding solvent contact surface has no self intersection.

\subsection{Convex Patches}

The convex patches of the solvent contact surface are exactly the same of the rolling ball surface. This derives immediately from the following:

Lemma 3 The solvent contact surface $S_{c}$ of the molecule $\mathcal{B}$ is completely included within the region between $\partial \mathcal{B}$ and $\partial \mathcal{B}^{\prime}$, where $\partial \mathcal{B}^{\prime}$ is the corresponding solvent accessible surface.

Since $S_{c}$ does not intersect the interior of $\mathcal{B}$ there is no nee to further trim the convex patches since they belong to $\partial \mathcal{B}$.

\subsection{Toroidal Patches}

First of all, we exclude the possibilities of two toroidal patches intersecting each other and of a toroidal patch intersecting with a concave/convex patch.

Lemma 4 Given two toroidal patches $T_{i}, T_{j}$ (with $i \neq j)$ their relative interiors are disjoint:

$$
\stackrel{\circ}{T}_{i} \cap \stackrel{\circ}{T}_{j}=\emptyset
$$

Lemma 5 Given a toroidal patch $T_{i}$ and a concave (convex) patch $C_{j}$, their relative interiors are disjoint:

$$
\stackrel{\circ}{T}_{i} \cap \stackrel{\circ}{C}_{j}=\emptyset
$$




\section{$\theta$}

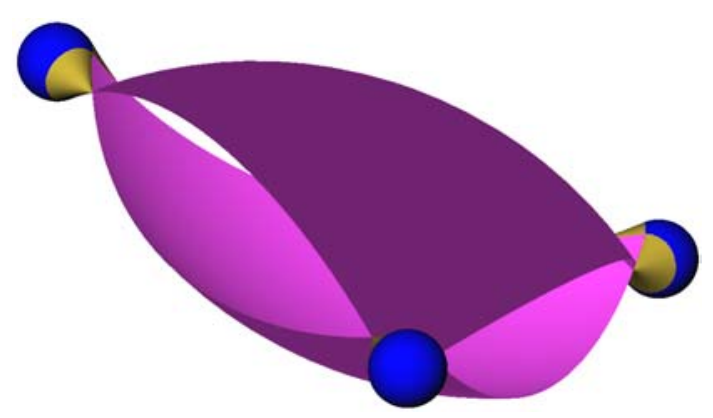

(a)
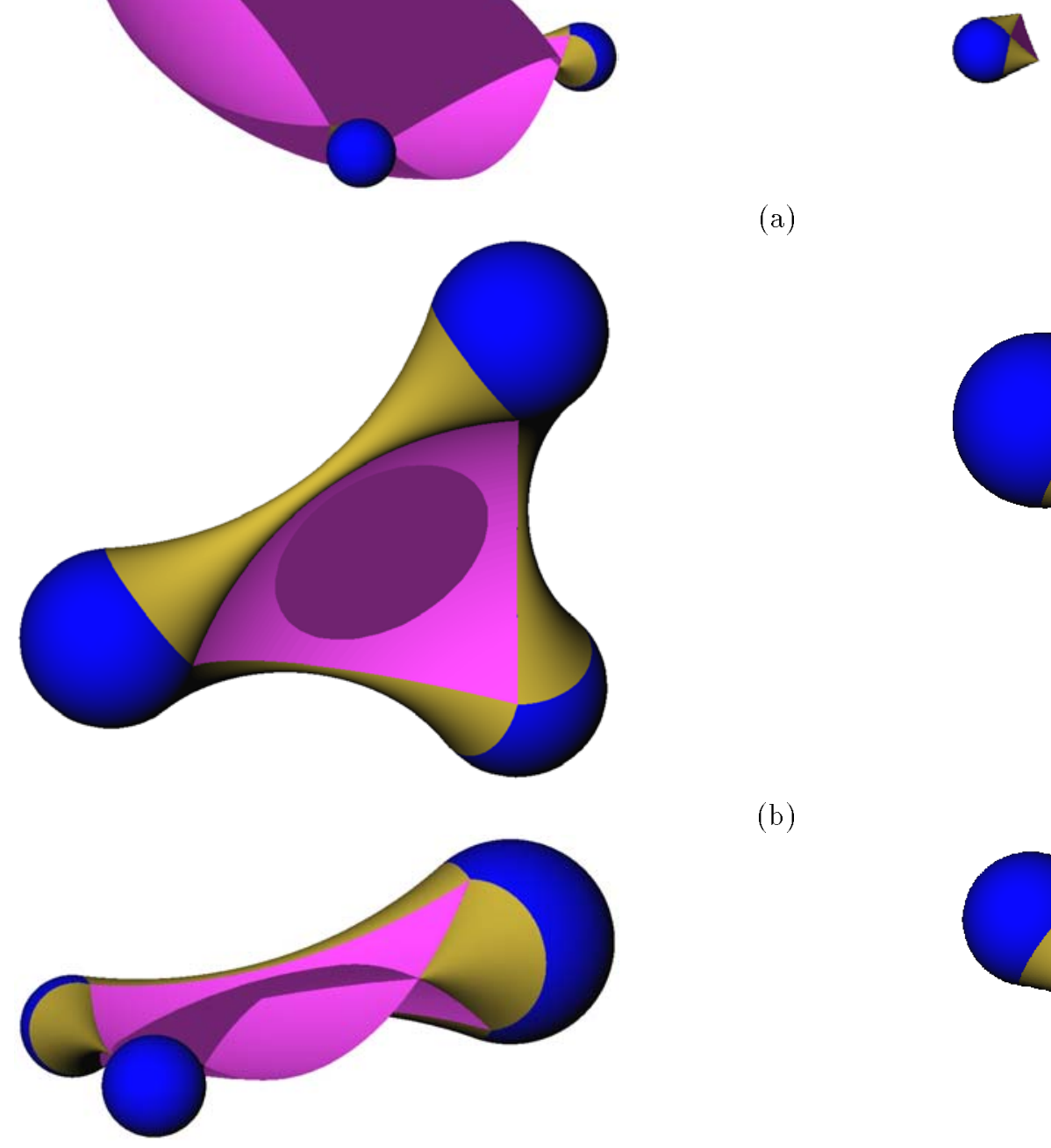

(b)
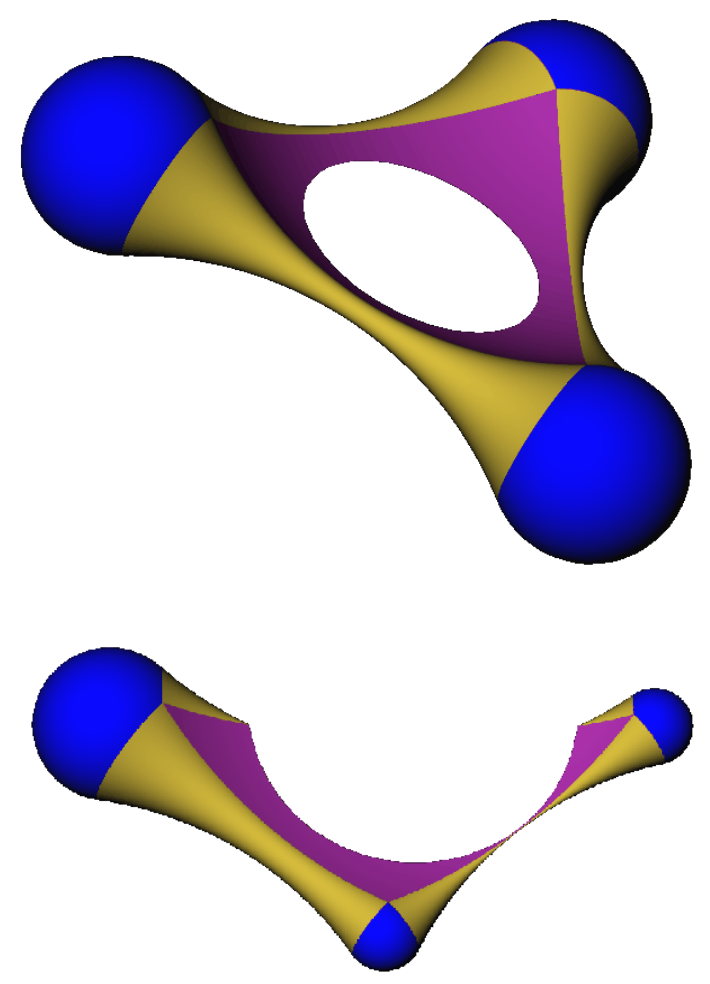

(c)

Figure 9: Three possible configurations of the solvent contact surfaces and rolling ball surfaces for different radii of the solvent and molecule atoms. On the left the self-intersecting rolling ball surfaces are shown. On the right the corresponding solvent contact surfaces are shown (without self-intersections). 
From the two previous lemmas we derive that one toroidal patch can intersect only itself. This happens when it can be constructed as rotational surface of an arc of circle around an axes that intersect the arc (see figure 10). For each arc a rotating around an axis $l$ intersecting $a$ we must remove that portion of $a$ lying on the "wrong" side of $l$. In this way we compute the arc $a^{\prime}$ (a disconnected subset of $a$ ) whose rotational surface around $l$ has no self intersection as in figure 8 .

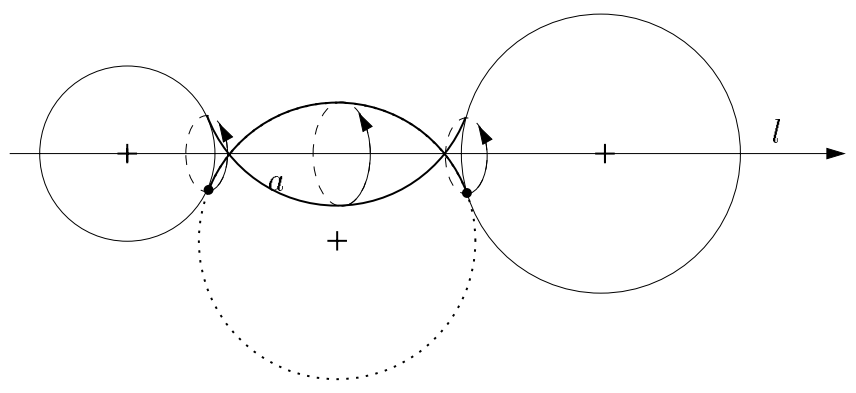

(a)

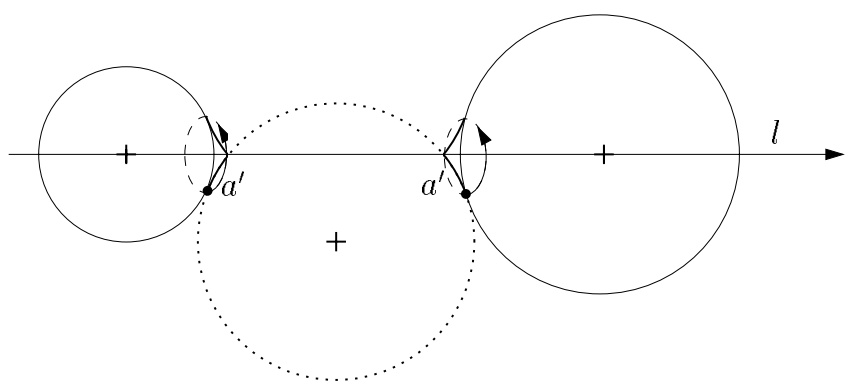

(b)

Figure 10: (a) The arc a rotating around the axes $l$ describes a self intersection portion of torus. (b) The arc $a^{\prime}$ rotating around the axes $l$ describes portion of torus with no self intersection.

\subsection{Trimming the Concave Patches}

First of all, we exclude the possibility of a concave patch intersecting either itself or a convex patch (we already know that it cannot intersect a toroidal patch).

Lemma 6 Given a concave patch $C_{i}$ and a convex patch $C_{j}$, their relative interiors are disjoint:

$$
\stackrel{\circ}{C}_{i} \cap \stackrel{\circ}{C}_{j}=\emptyset
$$

\section{Lemma 7 One concave patch cannot intersect itself.}

As show in Figure 9 two distinct concave patches can intersect each other. Since each concave patch is a portion of sphere we have to deal again with a sphere-sphere intersection problem. Hence we can simply maintain the regular triangulation of the centers of the concave patches (in this case all the weights are equal) so that we have all the relation of reciprocal intersection between concave patches. It has been shown in section 3 that the intersection between each pair of spheres is mapped to the insertion of an additional trimming circle in the domain space. Taking into account the intersections between pairs of concave patches, we must add some trimming circles to the domains of each concave patch to obtain the result of Figure 9.

\subsection{Correctness of the Result}

After the additional trimming curves are added to each toroidal/concave patch we get an exact representation of the solvent contact surface $S_{c}$ (see Figure 11).

Theorem 2 Any point in the solvent contact surface of the molecule $\mathcal{B}$ belongs to one of the patches of $S_{c}$ computed in the section above.

Proof: From Theorem 1 we are "locally" guaranteed that $S_{c}$ is the solvent contact surface of $\mathcal{B}$. Moreover the additional trimming curves guarantee that there is no legal ball $B^{\prime}$ (not only in the neighborhood of $B$ ) that contains in its interior any point $p \in \operatorname{cap} S_{r}$. Hence the theorem.

\section{Acknowledgments}

We would like to thank Herbert Edelsbrunner for the useful suggestions. This work has been supported in part by NSF grant CCR 9222467, AFOSR grant F49620-94-1-0080, ONR grant N00014-94-1-0370 and ARO grant DAAH04-95-1-0008.

\section{APPENDIX A - control points computation -}

In this appendix we explain the computation of the NURBS control points. The approach we take is to compute the control points once for all molecule atoms. That is each atom will be represented by its specific domain $D$ in $(u, v)$ space and the same set of normalized control points that represent the unitary sphere with center in the origin. Then we apply an affine transformation to map the unitary sphere to the position taken by the atom. To have a unique base set of control points (defining a portion of the normalized sphere) that can represent any atom we need to be sure that for each ball $B$ in $\mathcal{B}$ there is at least a neighbor ball $\bar{B}$, that intersects $B$ for the smallest portion. This is because we wish to compute the control points of a portion of 


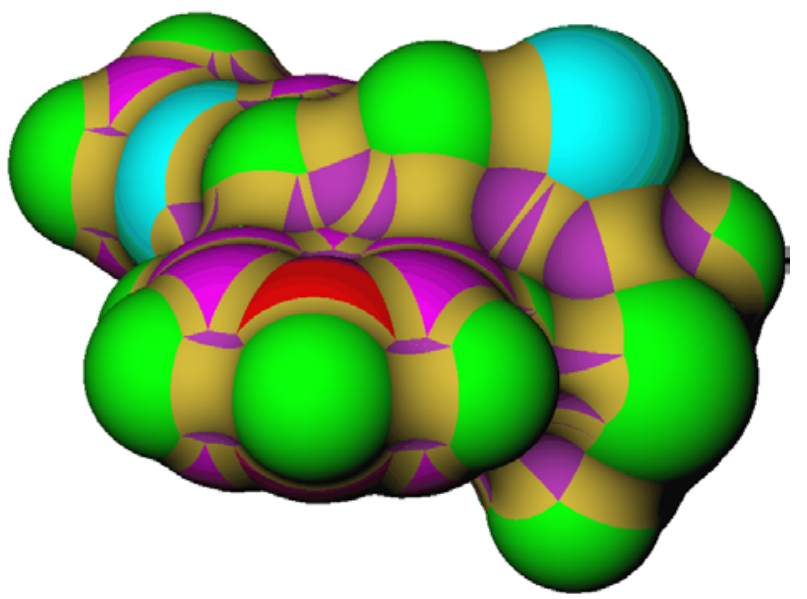

sphere which is a (bounded) rectangular domain and a minimum superset of any domain $D$ of any atom.

Fortunately this condition is satisfied for all molecules. For example in the ball and stick representation used in Raster3D [2, 16] a bond (stick) is drawn between to atoms of radii $r_{1}, r_{2}$ if the distance $r$ between the centers of the two atoms is less than $0.6\left(r_{1}+r_{2}\right)$. Since in a molecule there is at least one bond per atom we have that for each atom there is at least a neighbor atom for which $r<0.6\left(r_{1}+r_{2}\right)$. If we also consider that minimum atom size in a molecule is $1.3 \AA$ and the maximum is $2.18 \AA$ we have that each atom is intersected by a neighbor atom for at least $0.15477 \%$ of its radius. This means that, with reference to equation (2) we can always assume to have $d \leq 0.84523$ that is $l \leq 3.45288299571568$. For this fixed value of $l$ we apply a change of polynomial basis to get the coordinates $(x, y, z)$ of one quarter of the control points (and relative weight $w$ ) as in the table below.

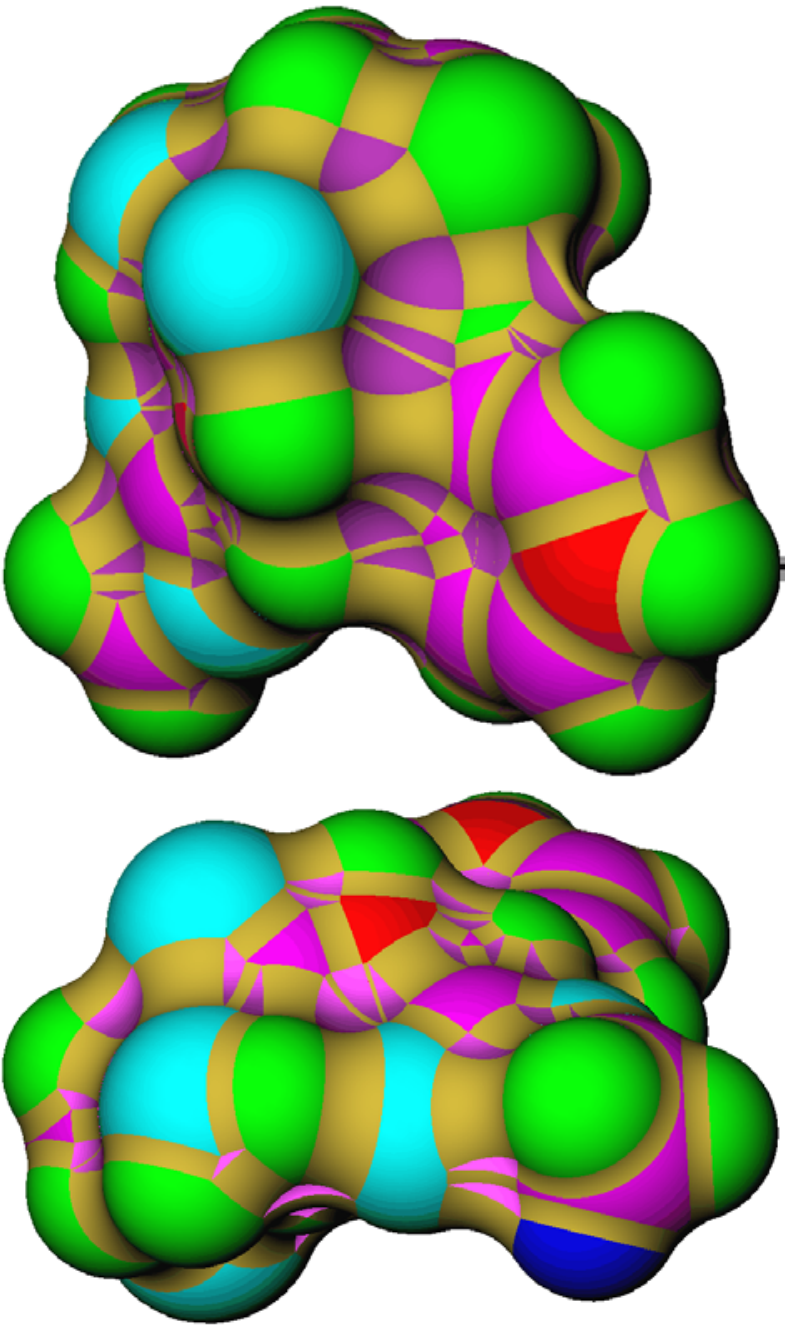

Figure 11: Complete Connolly surface of a Nutrasweet molecule.

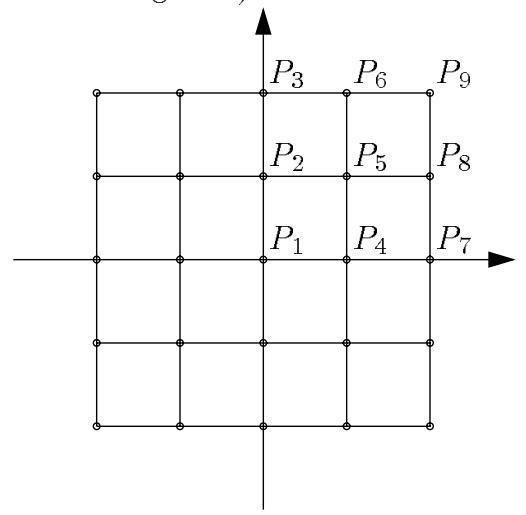

\begin{tabular}{|l|l|l|r|r|}
\hline & $x$ & $y$ & $z$ & $w$ \\
\hline$P_{1}$ & 0 & 0 & -1 & 1 \\
\hline$P_{2}$ & 0 & 1 & -1 & 1 \\
\hline$P_{3}$ & 0 & 2 & 0 & 2 \\
\hline$P_{4}$ & 1 & 0 & -1 & 1 \\
\hline$P_{5}$ & 1 & 1 & -1 & 1 \\
\hline$P_{6}$ & 1 & 2 & 0 & 2 \\
\hline$P_{7}$ & 2 & 0 & 0 & 2 \\
\hline$P_{8}$ & 2 & 1 & 0 & 2 \\
\hline$P_{9}$ & 2 & 2 & 1 & 3 \\
\hline
\end{tabular}

The other control points are just computed mirroring these twice with respect to the $x$ and $y$ axis. The knots vectors are $u:[-1-1-100111] v:[-1-1-100111]$.

\section{References}

[1] Aurenhammer, F. Voronoi diagrams - a survey of a fundamental geometric data structure. $A C M$ Comput. Surveys 23 (1991), 345-406.

[2] Bacon, D. J., and Anderson, W. F. A fast algorithm for rendering space-filling molecule pic- 
tures. Journal of Molecular Graphics 6 (1988), 219-220.

[3] Bajaj, C., and Kim, M. Generation of configuration space obstacles: The case of moving algebraic curves. Algoritmica \& (1989), 157-172.

[4] Bajaj, C., and Royappa, A. Finite representations of real parametric curves and surfaces. Internat. J. Comput. Geom. Appl. 5, 3 (1995), 313-326.

[5] Bajaj, C. L., and Bouma, W. J. Dynamic Voronoi diagrams and Delaunay triangulations. In Proc. 2nd Canad. Conf. Comput. Geom. (1990), pp. $273-277$.

[6] Branden, C., And Tooze, J. Introduction to Protein Structure. Garland Publishing Inc., 1991.

[7] Connoliy, M. L. Analytical molecular surface calculation. J. Appl. Cryst. 16 (1983), 548-558.

[8] Connolly, M. L. Solvent-accessible surfaces of proteins and nucleic acids. Science 221 (1983), 709-713.

[9] Edelsbrunner, H. The union of balls and its dual shape. Discrete Comput. Geom. 13, 3-4 (1995), $415-440$.

[10] Edelsbrunner, H., Facello, M., and J.Liang. On the definition and the construction of pockets in macromolecules. Report UIUCDCS-R-95-1935, University of Illinois Urbana-Champaign, 1995.

[11] Edelsbrunner, H., and Mücke, E. P. Three dimensional alpha shapes. ACM Transaction on Graphics 13, 1 (1994), 43-72.

[12] Fletterick, R. J. Molecular Structure: macromolecules in three dimensions. Blackwell Scientific publications, 1985 .

[13] Fu, J.- J., AND LEE, R. C. T. Voronoi diagrams of moving points in the plane. Internat. J. Comput. Geom. Appl. 1, 1 (1991), 23-32.

[14] Imai, H., Iri, M., and Murota, K. Voronoi diagram in the laguerre geometry and its applications. SIAM J. Comput. 14 (1985), 93-10.

[15] Krishnan, S., Manocha, D., and Narkhede, A. Representation and evaluation of boolean combinations of nurbs solids. In Fifth MSIStony Brook Workshop on Computational Geometry, Stony Brook (October 20-21 1995).

[16] Merritt, E. A., And Murphy, M. E. P. Raster3d version 2.0: A program for photorealistic molecular graphics. Acta Cryst. D50 (1994), $869-873$.
[17] Piegl, L., and Tiller, W. Curve and surface construction using rational b-splines. ComputerAided Design 19, 9 (1987), 485-498.

[18] Wernecke, J. The Inventor Mentor. AddisonWesley, 1994. 